\title{
Simulation of Crack Generation on a Concrete Wall
}

\author{
$\underline{\text { N. Mukai }}^{\text {a }}$, S. Fujita ${ }^{a}$ and Y. Chang ${ }^{a}$ \\ ${ }^{a}$ Graduate School of Engineering, Tokyo City University \\ Email: mukai@cs.tcu.ac.jp
}

\begin{abstract}
One of the applications using computer graphics is landscape simulation, which evaluates the scenery changed by constructing new buildings. The scenery is built with models generated by computer graphics modelling software or image based modelling techniques. The models generated by computer graphics modelling software is beautiful without stains or cracks on the building walls so that some sceneries are built by mapping real images of real buildings onto the walls of the buildings in order to improve the reality. However, even if real images are mapped onto the building walls, the scenery remains the same unless the images change as time passes. Many researchers have been trying to represent stains or cracks on the surfaces of objects built in a virtual space.
\end{abstract}

Cracks on buildings or roads affect the landscape simulation very much so that this paper considers how cracks are generated and how to represent them. A crack is generated by fatigue of materials that construct objects such as buildings or roads in a virtual space, and also the heat at the vertex of a crack affects the process of crack generation. In order to represent cracks on building walls, it is necessary to simulate the process of crack generation, and there are many methods for the simulation. The simplest method is the Mass-Spring model that has some vertices having masses, which are located at several points on the target material, and are connected with springs. FEM (Finite Element Method) is also generally used in the simulation, which divides the target material into finite small elements and calculates the stress in the material. These physically based simulations can generate detailed crack patterns; however, it takes a lot of time for the simulation so that some patterns are generated using leaf venation or Voronoi diagram. In addition, some patterns can be generated by NPR (NonPhotorealistic Rendering).

Previous works generated many kinds of crack patterns; however, those were generated on free surfaces on an object and stresses caused by the surrounding materials were not considered. In fact, a concrete wall that constructs a building is surrounded by reinforcing bars, and the crack is generated by the stress due to the reinforcing bars in addition to the gravity. Previous researches also did not consider the change of crack width. The crack can be elongated as time elapses; however, the width did not change even if time passed. Therefore, this paper proposes a simulation method of crack generated on a reinforced-concrete wall with physically based simulation, which method changes the width of crack as time passes. In this paper, we adopt RBSM (Rigid Body Spring Model) for the simulation, which can calculate the inner stress faster than FEM and generate more precise patterns than Mass-Spring model. In addition, we use Delaunay triangulation to generate a variety of crack patterns with small number of elements, and change the crack width by referring to the experimental results of crack on a reinforced-concrete wall.

As the result of the simulation, we have succeeded in generating crack patterns on a reinforced-concrete wall, where the crack grew with the width change as time passed. Figure 1 shows the result of the simulation and a real concreate wall. By comparing them, we can see that some parts surrounded by circles are similar.

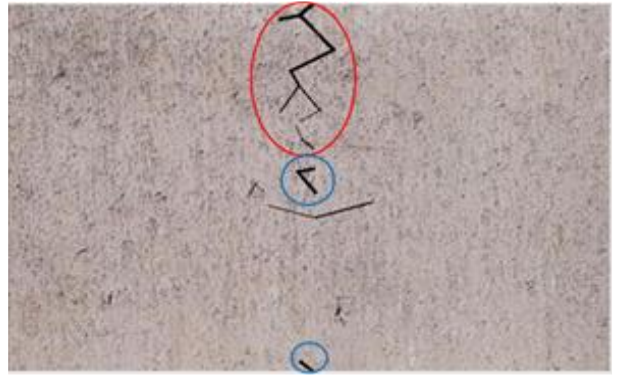

(a) simulation result

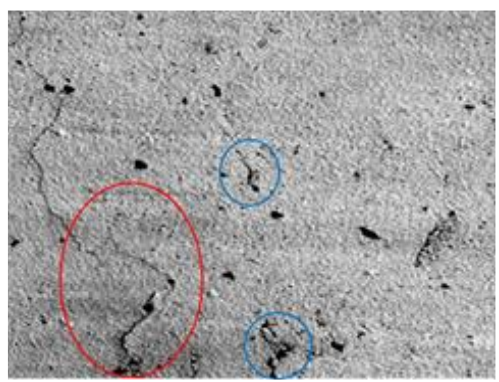

(b) real concrete

Figure 1. Comparison between a simulation result and a real concrete.

Keywords: Physically based simulation, crack generation, RBSM, Delaunay triangulation 


\section{INTRODUCTION}

Computer graphics is widely used for a variety of applications such as industrial design, commercial films, moves, games, simulations in a virtual space, simulation based visualizations, and so on. One of them is landscape simulation, which is a simulation in a virtual space, and is used for an evaluation of the scenery that is changed by constructing new buildings. The scenery is built with computer graphics models, which are generated using modelling software or image based modelling. The model generated by computer graphics modelling software has no stains or cracks so that some images are mapped onto the model surfaces to make the models realistic; however, it is not real unless the stains or cracks change as time passes. Then, many researches have been studied to investigate the process of crack generation and also to represent cracks on the surfaces of target objects.

Crack is generated by fatigue of materials so that the process of fatigue fracture was investigated by subdividing the process into 3 stages: crack generation, crack growth, and spontaneous development (e.g. Pokhmurskil et al., 1975). Fatigue crack is also affected by the temperature at the crack vertex so that the spatial and temporal variations of temperature at a crack vertex were investigated (e.g. Terekhina et al. 2012). In addition, crack generation mechanism was researched and it was found that amorphous silicon films were deformed convexly during heating and concavely after cooling and cracks were generated not during heating but during cooling (e.g. Tanaka et al. 2015).

On the other hand, there have also been many researches on generating crack patterns. Most researches are based on physically based simulation, and the simplest method is Mass-Spring model (e.g. Hirota et al. 1998). In order to represent detailed crack patterns, the inner stress that causes to generate cracks should be considered, and FEM is usually used (e.g. Moës et al. 1999). Physically based simulation considering inner stress of the target material can generate precise crack patterns and also can represent the process of the crack generation as an animation; however, the method requires a lot of time, for example 2.7 hours computation time for 75,000 triangles that construct an object (e.g. Iben et al. 2006). Then some researches use RBSM, which divides the target material into small pieces and uses spring model to analyze the behavior (e.g. Kawai 1978). There are also some derivations of RBSM such as RBSN (Rigid Body Spring Network)(e.g. Bolander et al. 2001) or 3D RBSM (e.g. Nagai et al. 2005).

In addition, there are another method that uses similar patterns such as leaf venation. By using leaf venation patterns and by inputting some parameters interactively, 2D crack patterns can be generated in real time (e.g. Colleoni Couto et al. 2010). There are also some studies that utilize Voronoi diagram (e.g. Schvartzman et.al. 2014) or Delaunay triangulation (e.g. kambayashi et al. 2014) to generate natural crack patterns. In addition, image based techniques are used to generate crack patterns such as Distance Transform algorithm for simulating the cracks in Batik wax painting (e.g. Wyvill et al. 2004) and image filter that transforms a line drawing to a fractured surface image (e.g. Mould 2005).

As mentioned above, there have been many studies related to the generation of crack patterns. Physically based simulation can generate precise and a variety of crack patterns and animate the process; however, it takes a lot of time to simulate. On the other hand, image based technique including utilization of leaf venation can generate crack patterns interactively although there is no guarantee of accuracy for the generated images. In addition, the crack patterns were generated on free surfaces and the conditions surrounding the target material was not considered. For instance, a concrete wall used for a building is surrounded by reinforcing bars and the crack is generated by the effect of the reinforcing bars and the gravity. In previous works, generated cracks were elongated as they grew; however, the width did not change even if time passed. Therefore, this paper proposes a physically based simulation method that generates crack patterns on a concrete surrounded by reinforcing bars, and considers that the width of crack changes as time passes by referring to an investigation result. 


\section{METHOD}

\subsection{Material selection}

In this paper, we generate crack patterns on a concrete wall; however, there are many types of concrete so that we have to specify the target concrete to simulate the crack generation process caused by stress. Table 1 shows several kinds of concretes. In Table 1 , general concrete is widely used for housing. In addition, our final goal is landscape simulation, where many houses are located and which can evaluate the scenery changed by new houses, so that we select general concrete for the simulation and apply the proposed method to a concrete wall surrounding a house.

On the other hand, there are also a variety of cracks. Table 2 shows the relation between crack and its primary factor. In Table 2, crack by shrinking is the most popular crack and it is often seen on house walls. In landscape simulation, cracks generated on surfaces of house walls have large impact to the scenery and we investigate the process of crack generation by calculating the inner stress of a general concrete. Then, we select crack by shrinking as the target crack.

\subsection{RBSM model}

We use RBSM as the simulation model since Mass-Spring model cannot simulate the inner stress of the material and FEM requires a lot of time to simulate. RBSM can consider the inner stress and the simulation is very fast because the calculation is based on spring model. Figure 2 shows 2 elements of RBSM and springs that connect the 2 elements. As Figure 2 shows, there are 2 springs on the boundary of the 2 elements: shear and vertical springs. If the stress calculated with RBSM exceeds the yield stress that is the strength of spring in RBSM model, the spring is broken and crack is generated.

\subsection{Delaunay triangulation}

In order to calculate the inner stress with RBSM, the target concrete surface should be divided into small elements. The simplest one is the grid-based division; however, we have to divide the target area into huge number of small elements to represent natural cracks, and it takes huge time to simulate. On the other hand, there is a division method called Voronoi diagram, which makes natural division with small number of elements. Figure 3 shows Voronoi diagram, where each element is composed of a area surrounded by perpendicular bisectors of generatrices, which are occurred at random. Voronoi diagram can generate a variety of shape with small and large areas; however, the vertex has only 3 line segments

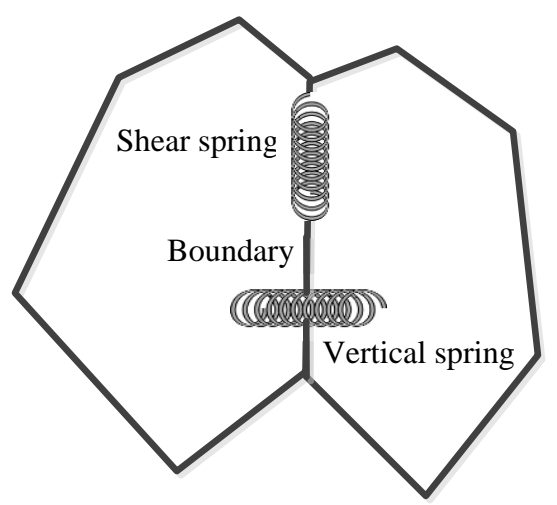

Figure 2. RBSM model.

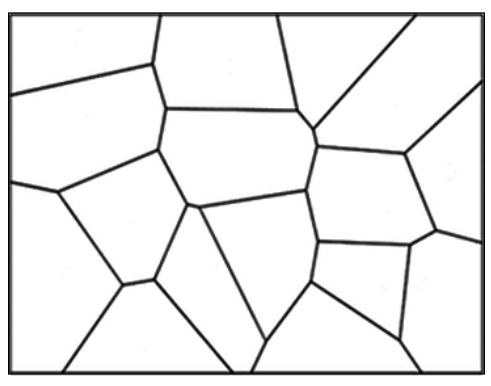

Figure 3. Voronoi diagram. 
as shown in Figure 3 so that the direction of crack is limited and the generated image of crack is unnatural. Then, we adopt Delaunay triangulation instead of Voronoi diagram for the division of the target area.

In this paper, we use Delaunay triangulation for the element division. The red triangles in Figure 4 shows Delaunay triangulation generated from another Voronoi diagram. As Figure 4 shows, the vertex has many line segments so that there exist some selections for the growing direction of a crack. The original Delaunay triangulation has no triangle along the surrounding edges. Then, some triangles should be made by connecting the vertices of the original Delaunay triangulation and 4 vertices of the rectangle or the intersection points of the perpendicular bisectors of generatices in Voronoi diagram and the edges of the rectangle. Figure 5 shows the Delaunay triangulation generated by this method.

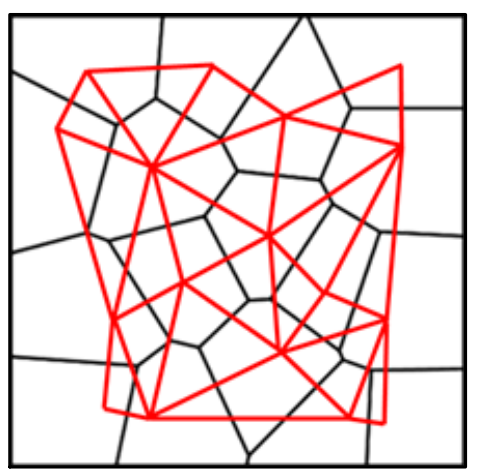

Figure 4. Relation between Voronoi diagram and Delaunay triangulation.

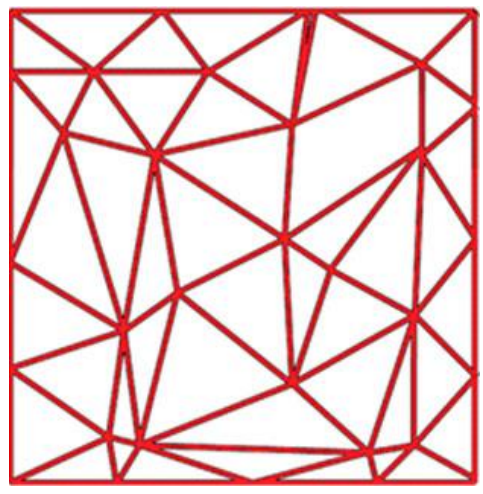

Figure 5. Delaunay triangulation.

\subsection{Constraint condition}

Our purpose is to generate cracks on a house wall made from a general reinforced-concrete. Then, the wall is constrained by reinforcing bars and the ground so that the force generated by the constraint occurs and is directed to the constraint objects: two reinforcing bars and the ground. Figure 6 shows the constraint and the force direction. We consider that some forces are loaded at some elements by the constraint. In Figure 6 , there are two reinforcing bars at the both sides of the wall and there is the ground at the bottom. We also consider cracks caused by shrinking so that the force directions on the right and left half sides are right and left, respectively. In addition, the force is also directed to the bottom due to the gravity.

\section{SIMULATION}

\subsection{Stiffness matrix}

The relation between inner stresses and their strains is described with the following equation.

$$
\sigma=K \varepsilon
$$

Where, $\mathbf{K}$ is stiffness matrix. For two elements in 2 dimension, Eq. (1) is represented as Eq.(2).

$$
\left[\begin{array}{l}
\sigma_{x}^{1} \\
\sigma_{y}^{1} \\
\sigma_{x}^{2} \\
\sigma_{y}^{2}
\end{array}\right]=\left[\begin{array}{c}
K_{x 1}^{x 1} K_{y 1}^{x 1} K_{x 2}^{x 1} K_{y 2}^{x 1} \\
K_{x 1}^{y 1} K_{y 1}^{y 1} K_{x 2}^{y 1} K_{y 2}^{y 1} \\
K_{x 1}^{x 2} K_{y 1}^{x 2} K_{x 2}^{x 2} K_{y 2}^{x 2} \\
K_{x 1}^{y 2} K_{y 1}^{y 2} K_{x 2}^{y 2} K_{y 2}^{y 2}
\end{array}\right]\left[\begin{array}{l}
\varepsilon_{x}^{1} \\
\varepsilon_{y}^{1} \\
\varepsilon_{x}^{2} \\
\varepsilon_{y}^{2}
\end{array}\right]
$$

Where, $\sigma_{j}^{i}$ represents the $\mathrm{j}$ directional stress of element $\mathrm{i}$, $\varepsilon_{j}^{i}$ represents the $\mathrm{j}$ directional strain of element $\mathrm{i}$, and $K_{j}^{i}$ represents the $\mathrm{j}$ directional stiffness for $\mathrm{i}$ directional strain. $\mathbf{K}$ is symmetry and also calculated as follows.

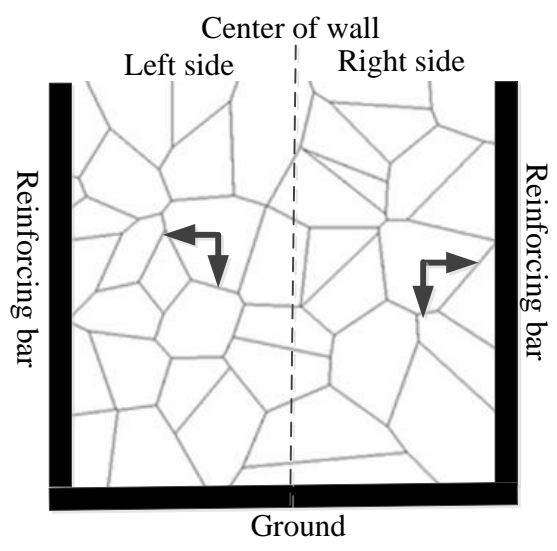

Figure 6. Constraints and force directions. 


$$
\begin{aligned}
& K_{x 1}^{x 1}=-k_{n} l_{y}^{2}+-k_{s} l_{x}^{2}, \quad K_{y 1}^{x 1}=-\left(k_{n}-k_{s}\right) l_{x} l_{y}, \quad K_{x 2}^{x 1}=-K_{x 1}^{x 1}, \quad K_{y 2}^{x 1}=-K_{y 1}^{x 1}, \\
& K_{y 1}^{y 1}=k_{n} l_{x}^{2}+-k_{s} l_{y}^{2}, \quad K_{x 2}^{y 1}=-K_{y 1}^{x 1}, \quad K_{y 2}^{y 1}=-K_{y 1}^{y 1}, \\
& K_{x 2}^{x 2}=K_{x 1}^{x 1}, \quad K_{y 2}^{x 2}=K_{y 1}^{x 1}, \quad K_{y 2}^{y 2}=K_{y 1}^{y 1} \ldots \ldots \ldots \ldots \ldots \ldots \ldots \ldots \ldots \ldots \ldots \ldots \ldots \ldots \ldots \ldots \ldots \ldots \ldots \ldots \ldots \ldots \ldots \ldots \ldots \ldots \ldots \ldots \ldots \ldots \ldots \ldots \ldots \ldots \ldots \ldots \ldots
\end{aligned}
$$

Where, $k_{n}$ and $k_{s}$ are vertical and shear elements of spring constant, respectively, and $l_{x}$ and $l_{y}$ are $x$ and y directional length of the boundary between the element 1 and 2 , respectively. $k_{n}$ and $k_{s}$ are also calculated with the following equation.

$$
k_{n}=\frac{E}{\left(1-v^{2}\right)\left(L_{1}+L_{2}\right)}, \quad k_{S}=\frac{E}{(1+v)\left(L_{1}+L_{2}\right)}
$$

Where, $E$ is Young's modulus, $v$ is Poisson's ratio, and $L_{1}$ and $L_{2}$ are lengths from the centers of gravity of element 1 and 2 to the boundary, respectively.

Actually, each strain can be calculated from forces that are loaded at some elements with the similar equation. Then, we can calculate the every inner stress from each strain by generating the stiffness matrix for all elements. Finally, when the inner stress is over the yield stress, the spring is broken and a crack is generated. In RBSM, each element has 2 springs: vertical and shear. Then, when at least one of the springs is broken, a crack is generated.

\subsection{Crack width}

As a crack grows, its width changes, and it actually shrinks after it has swelled, and this process is repeated. The speed of this process is different for each material, and depends on the final value of drying shrinkage strain. The maximum width of crack, however, is the same for every material even if the final value of drying shrinkage strain is different (e.g. Seo et al. 2005). In this paper, we change the crack width according to the experimental result related to the relation between the concrete age and the crack width.

\section{RESULT}

We have simulated the process of crack generation with the proposed method for 500 elements on the image, which resolution is $600 \times 400$. Delaunay triangulation used in the simulation is shown in Figure 7. Two types of the simulation results are shown in Figure 8, where a real image that has no crack is mapped as the background image.

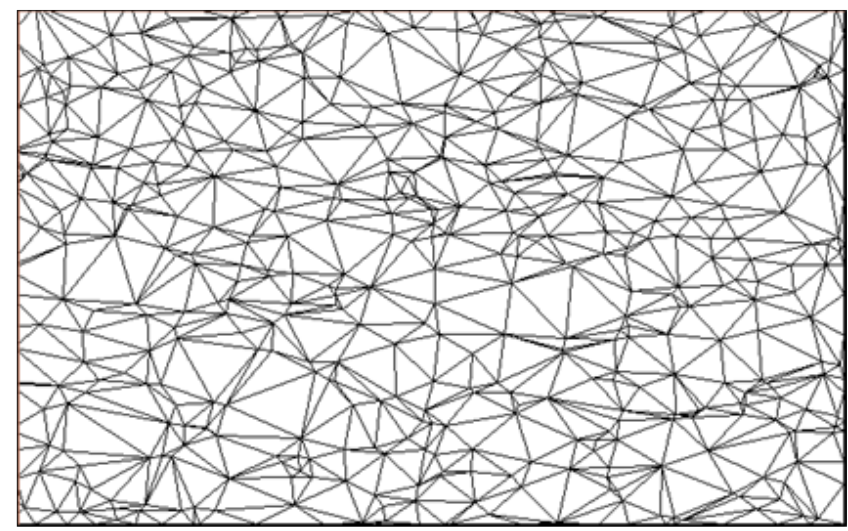

Figure 7. Delaunay triangulation used for the simulation. 
The concreate is constrained with 2 reinforcing bars for the both sides and the ground for the bottom, so that some forces are loaded by the shrinking. Then, some strains are generated by the forces, and cracks are generated when the inner stress is over the yield stress. In Figure 8, it seems that the horizontal stress is larger than the vertical one so that the crack runs vertically. As time passes, cracks grow changing their widths; however, the width does not grow linearly but swelling and shrinking are repeated alternately. In Figure 8, there are two types of simulation results: $600 \mu$ and $400 \mu$ for the final values of drying shrinkage strain. By comparing the 2 results, we can see that the concrete with $600 \mu$ final value of drying shrinkage strain grows faster than the other concrete with $400 \mu$ final value of drying shrinkage strain, and has more cracks. However, the maximum width of the cracks is the same for the both simulation results although the width changes during the process of crack generation.

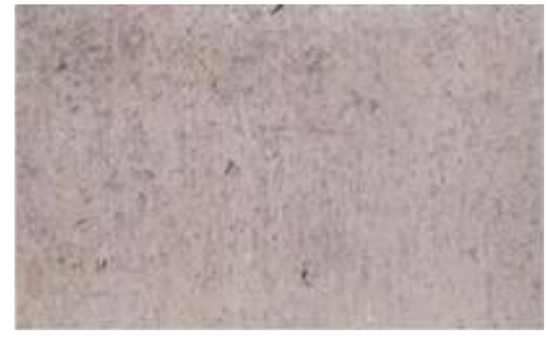

(a1) after 2 months

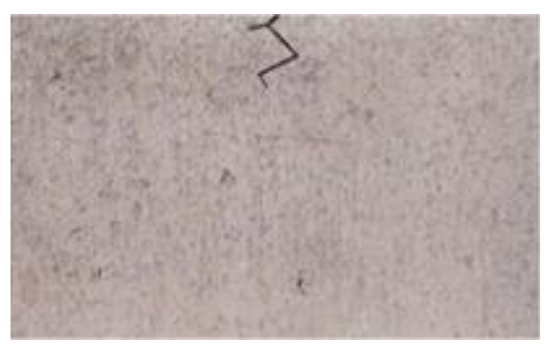

(a2) after 6 months

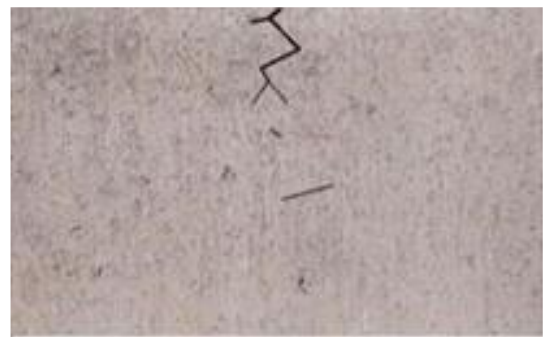

(a3) after 18 months

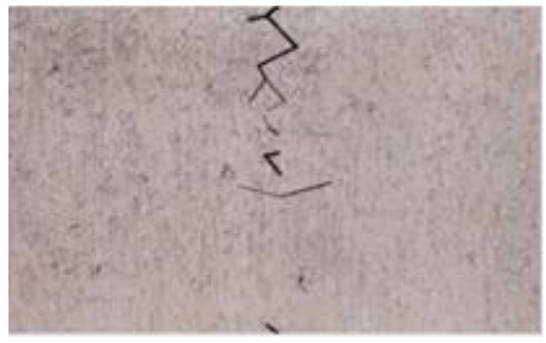

(a4) after 24 months

(a) $400 \mu$

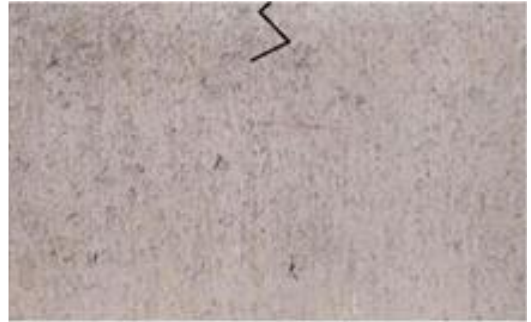

(b1) after 2 months

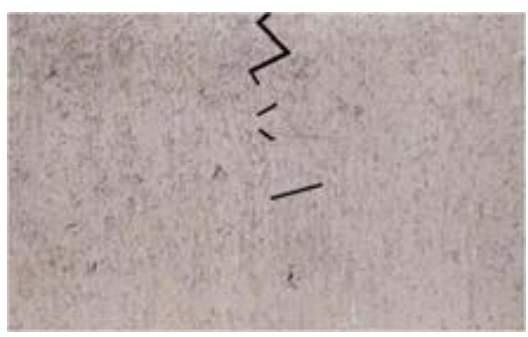

(b2) after 6 months

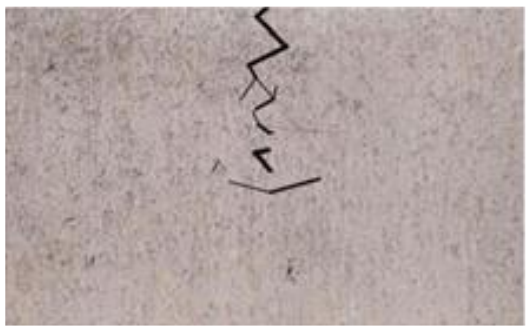

(b3) after 18 months

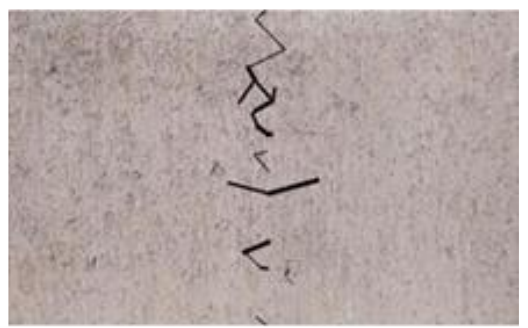

(b4) after 24 months

(b) $600 \mu$

Figure 8. Simulation results for two types of final values of drying shrinkage strain.

\section{CONCLUSIONS AND FUTURE WORKS}

In this paper, we have proposed a crack generation method using RBSM, which considers the constraints from the reinforcing bars and the ground, and also the change of crack width. For the precise simulation, huge number of elements are required so that we have adopted Delaunay triangulation to divide the target area into 
some simulation elements for natural division. As the results of the simulation, crack runs vertically due to the constraints from the reinforcing bars and the ground, and the width changed as time passed. By comparison between the concrete with $600 \mu$ of final value of drying shrinkage strain and another concrete with $400 \mu$ of final value of drying shrinkage strain, we have found that crack on the concrete with $600 \mu$ of final value of drying shrinkage strain grows faster than the other concrete. In addition, we have confirmed that the cracks generated by the simulation is similar to the real one by comparing the simulation result with a real concrete as Figure 1 shows.We have succeeded in visualizing the process of the crack generation; however, the width of crack is uniform along the boundary of elements although it changes as time passes. In the real crack, however, the width is not uniform. In addition, the shape of the crack depends on the Delaunay triangulation, which is defined by the generatrices used for Voronoi diagram. Then, we also have to consider how to define the location of generatices as the future works.

\section{REFERENCES}

Bolander, J.E., Yip, M., Moriizumi, K., and Kunieda, M. (2001). Rigid-body-spring network of cement-based composites. Fracture Mechanics of Concrete Structures, de Borst el al.(eds), 773-780.

Colleoni Couto, J.M., Marcelino, T., Braun, H., Strube de Lima, D., and Raupp Musse, S. (2010). Generation of cartoon 2D cracks based on leaf venation patterns. Proceesings of the Brazilian Symposium on Games and Digital Entertainment, 165-170.

Hirota, K., Tanoue, Y., and Kaneko, T. (1998). Generation of crack patterns with a physical model. The virtual Computer, 14(3), 126-137.

Iben, H.N., and O'Brien, H.F. (2006). Generating surface crack patterns. Proceedings of the ACM SIGGRAPH/Eurographics Symposium on Computer Animation, 177-185.

Kambayashi, A., Fujiwara, Y., Takeuchi, N., and Shiomi, T. (2014). Discrete crack analysis of RC structure using hybrid-type penalty method with Delaunay triangulation. $11^{\text {th }}$ World Congress on Computational Mechanics, 1-12.

Kawai, T. (1978). New discrete models and their application to seismic response analysis of structures. Nuclear Engineering and Design, ELSEVIER, 48(1), 207-229.

Moës, N., Dolbow, J., and Belytchko, T. A. (1999). Finite element method for crack growth without remeshing. International Journal for Numerical Mehtods in Engineering, 46, 131-150.

Mould, D. (2005). Image-guided fracture. Proceedings of Graphics Interface, 219-226.

Nagai, K., Sato, Y., and Ueda, T. (2005). Mesoscopic simulation of failure of mortar concrete by 3D RBSM. Journal of Advanced Concrete Technology, 3(3), 385-402.

Pokhmurskil, V.I., Levitskil, M.O., and Mikitishin, S.I. (1975). Time to fatigue-crack generation and speed of fatigue-crack growth in steels 08kp and U8. Translated from Fiziko-khimicheskaya Mekhanka Materialov, 11(4), 41-44.

Schvartzman, S.C., and Otaduy, M.A. (2014). Fracture animation based on high-dimensional voronoi diagrams. Proceedings of the $18^{\text {th }}$ meeting of the ACM SIGGRAPH Symposium on Interactive 3D Graphics and Games, $15-22$.

Seo, T., Ohno, Y., and Nakagawa, T. (2005). Influence of concrete strength and steel ratio on cracking due to drying shrinkage in RC wall. Proceedings of the annual conference in Architectural Institute of Japan, 641642.

Tanaka, K., Hayashi, S., Morisaki, S., and Higashi, S. (2015). Investigations on crack generation mechanism and crack reduction by buffer layer insertion in thermal-plasma-jet crystallization of amorphous silicon films on glass substrate. Japanese Journal of Applied Physics, 54, 01 AE05 1-5.

Terekhina, A.I., Bannikov, M.V., Plekhov, O.A., and Plekhova, E.V. (2012). Experimental study of heat generation at the vertex of a fatigue crack. Technical Physics Letters, 38(8), 736-738.

Wyvill, B., Overveld, K.V., and Carpendale, S. (2004). Rendering cracks in batik. Proceedings of the $3^{\text {rd }}$ International Symposium on Non-photorealistic Animation and Rendering, 61-149. 